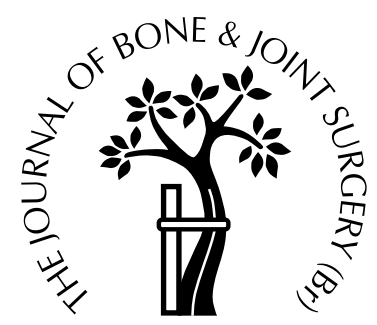

\title{
Proprioception after rehabilitation and reconstruction in knees with deficiency of the anterior cruciate ligament
}

\author{
A PROSPECTIVE, LONGITUDINAL STUDY \\ R. W. Fremerey, P. Lobenhoffer, J. Zeichen, M. Skutek, U. Bosch, \\ H. Tscherne \\ From Hannover Medical School, Hannover, Germany
}

$\mathbf{W}$ e assessed proprioception in the knee using the angle reproduction test in $\mathbf{2 0}$ healthy volunteers, ten patients with acute anterior instability and 20 patients with chronic anterior instability after reconstruction of the anterior cruciate ligament (ACL). In addition, the Lysholm-knee score, ligament laxity and patient satisfaction were determined.

Acute trauma causes extensive damage to proprioception which is not restored by rehabilitation alone. Three months after operation, there remained a slight decrease in proprioception compared with the preoperative recordings, but six months after reconstruction, restoration of proprioception was seen near full extension and full flexion. In the mid-range position, proprioception was not restored. At follow-up, $3.7 \pm 0.3$ years after reconstruction, there was further improvement of proprioception in the mid-range position. There was no difference between open and arthroscopic techniques. The highest correlation was found between proprioception and patient satisfaction. After reconstruction of the ACL reduced proprioception may explain the poor functional outcome in some patients, despite restoration of mechanical stability.

J Bone Joint Surg [Br] 2000;82-B:801-6.

Received 22 June 1999; Accepted after revision 14 January 2000

The complex kinematics of the knee depend on both mechanical stability and dynamic interaction between the central nervous system and the joint. ${ }^{1}$ Proprioception (Fig. 1) gives information about movement (kinaesthesia) and position (position sense) of the knee and is important in its muscular control. The afferent innervation is based on peripheral receptors in the joint, muscles and skin. ${ }^{2}$ Ruffini

R. W. Fremerey, MD, Trauma Surgeon

P. Lobenhoffer, MD, Trauma Surgeon

J. Zeichen, MD, Trauma Surgeon

M. Skutek, MD, Trauma Surgeon

U. Bosch, MD, Trauma Surgeon

H. Tscherne, MD, Professor

Trauma Department, Hannover Medical School, Carl-Neuberg Strasse 1, D30625 Hannover, Germany.

Correspondence should be sent to Dr R. W. Fremerey.

(C)2000 British Editorial Society of Bone and Joint Surgery

0301-620X/00/610306 \$2.00 endings, Pacinian corpuscles and Golgi tendon organs are mechanoreceptors which have been identified histologically in various intra-articular structures of the knee as have free nerve endings. ${ }^{3,4}$ Between $1 \%$ and $2 \%$ of the volume of the anterior cruciate ligament (ACL) consists of mechanoreceptors and therefore rupture of the ACL reduces proprioception. Most of the mechanoreceptors and free nerve endings are subsynovial, close to the femoral and tibial insertion of the ligament.

Reconstruction of the ACL can be achieved successfully, by a variety of procedures, but many authors have reported poor correlation between the clinical signs, knee assessment scores and function. Some patients who have persistent ligamentous laxity after reconstruction return to full activity without symptoms. Others, with a clinically satisfactory ACL reconstruction, good ligamentous stability and high clinical knee scores, continue to suffer from subjective instability and episodes of giving-way. These patients appear to have poor joint position sense caused by lack of proprioception. The pattern of proprioceptive recovery after ACL reconstruction is still not clear. ${ }^{1,5}$ We have therefore investigated the loss of proprioception after acute rupture of the ACL and analysed the potential for the return of proprioception by either intensive rehabilitation or reconstruction in a prospective, longitudinal study. The correlation between proprioception, patient satisfaction, the Lysholm knee score and ligamentous laxity was also assessed.

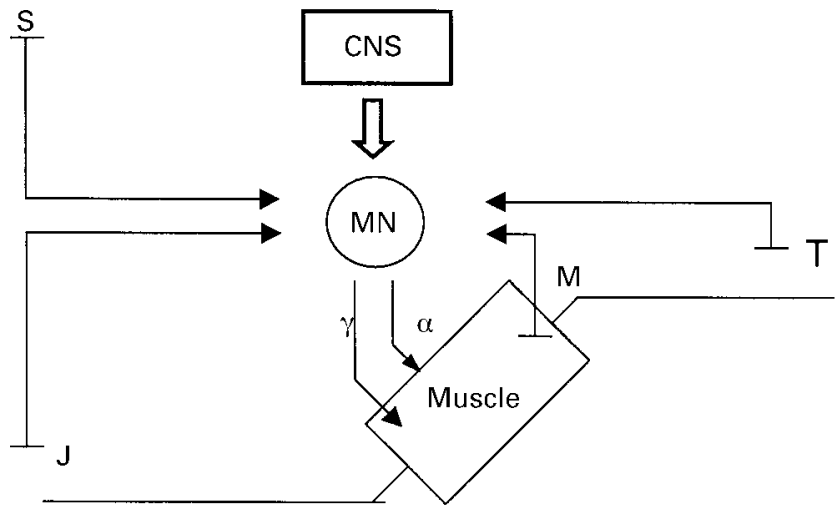

Fig. 1

Diagram showing the central control of the CNS and the peripheral control by the muscle-spindle system $(\mathrm{M})$, joint receptors $(\mathrm{J})$ and by mechanoreceptors located in the skin $(\mathrm{S})$ and tendons $(\mathrm{T})(\gamma$, gamma efferents; $\alpha$, alpha efferents; MN, motor neurone). 
R. W. FREMEREY, P. LOBENHOFFER, J. ZEICHEN, M. SKUTEK, U. BOSCH, H. TSCHERNE

Table I. Details of the 20 patients with chronic anterior knee stability who had reconstruction of the ACL

\begin{tabular}{|c|c|c|c|c|}
\hline & \multicolumn{4}{|c|}{ Technique used } \\
\hline & \multicolumn{2}{|c|}{$\begin{array}{l}\text { Arthroscopy } \\
(\mathbf{n}=\mathbf{1 1})\end{array}$} & \multicolumn{2}{|c|}{$\begin{array}{l}\text { Limited arthrotomy } \\
(\mathbf{n}=9)\end{array}$} \\
\hline & Male & Female & Male & Female \\
\hline Number of patients & 7 & 4 & 6 & 3 \\
\hline Mean ( \pm SD) age in years & $27.9 \pm 4.8$ & $26.8 \pm 3.9$ & $29.7 \pm 5.1$ & $28.9 \pm 3.7$ \\
\hline $\begin{array}{l}\text { Mean }( \pm \mathrm{SD}) \text { time from } \\
\text { trauma to reconstruction in months }\end{array}$ & $11.8 \pm 3.2$ & $13.9 \pm 5.1$ & $13.2 \pm 4.0$ & $10.9 \pm 2.6$ \\
\hline Tear medial meniscus & 1 & 2 & 2 & 0 \\
\hline Tear lateral meniscus & 1 & 0 & 2 & 1 \\
\hline Tear medial and lateral meniscus & 1 & 0 & 1 & 1 \\
\hline
\end{tabular}

\section{Patients and Methods}

Patients with acute anterior knee instability. We examined ten patients ( 7 men and 3 women, mean age $22.7 \pm 3.2$ years) with an acute rupture of the ACL within the first 12 days (mean $6.3 \pm 3.0$ ) of injury. There was no history of injury on the contralateral side. Seven patients required aspiration of a haemarthrosis. Treatment included the administration of non-steroidal anti-inflammatory drugs, non-weight-bearing and a rehabilitation programme. All knees were swollen and painful, and the diagnosis was later confirmed arthroscopically.

Patients with chronic anterior knee instability. There were 20 patients ( 7 women and 13 men, mean age $28.4 \pm$ 4.4 years) with chronic anterior knee instability who had undergone ACL reconstruction in our department between June 1995 and May 1996 at a mean of $12.4 \pm 3.7$ months after injury. They were investigated before and at three and six months after operation. The final examination included 18 of the 20 patients and was carried out after a mean follow-up of $3.7 \pm 0.3$ years ( 3.3 to 4.2 ). One patient had an additional knee injury on the contralateral side (rupture of the medial collateral ligament) and could not be tested, and one was lost to follow-up. The injury was caused during sporting activities, mostly soccer, in 16 patients $(80 \%)$, by road-traffic accidents in three (15\%) and by a fall from a ladder in one.

Before operation, all had attended a standardised rehabilitation programme. Instability had been confirmed by examination under anaesthesia and by arthroscopy. Four patients had a tear of the lateral meniscus, five had damaged the medial meniscus and three had a lesion of both menisci (Table I). The necessary meniscal surgery was carried out arthroscopically in all patients. None had a history or indication of a significant knee injury or disease of the contralateral side. The criteria for withdrawal from the investigation were evidence of rheumatoid or neurological disease, alcoholism, drug addiction and poor compliance.

Control group. A control group consisted of 20 healthy volunteers ( 7 women and 13 men) with a mean age of 26.4 \pm 4.8 years and a mean Tegner activity level of $5.5 \pm 1.1$

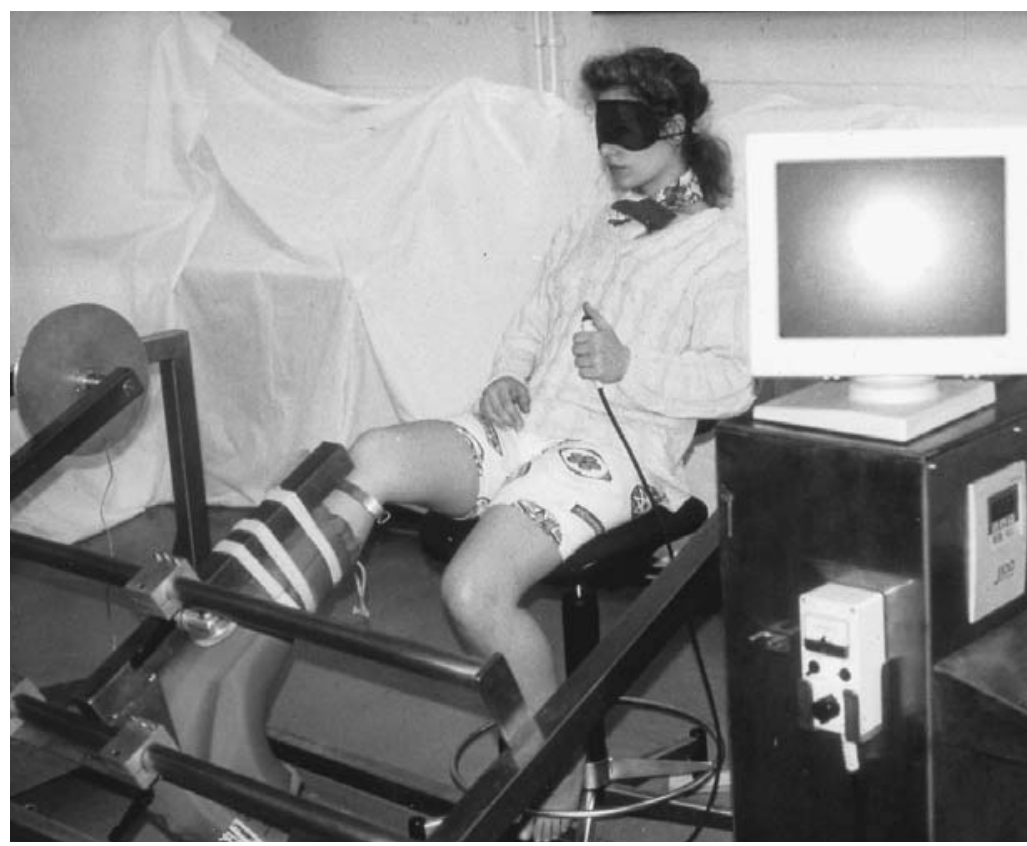

Fig. 2

Proprioception testing apparatus as suggested by Barrett. ${ }^{1}$ The reproduction of passive positioning was carried out at a constant angle velocity, with the patient blindfolded in a sitting position. 


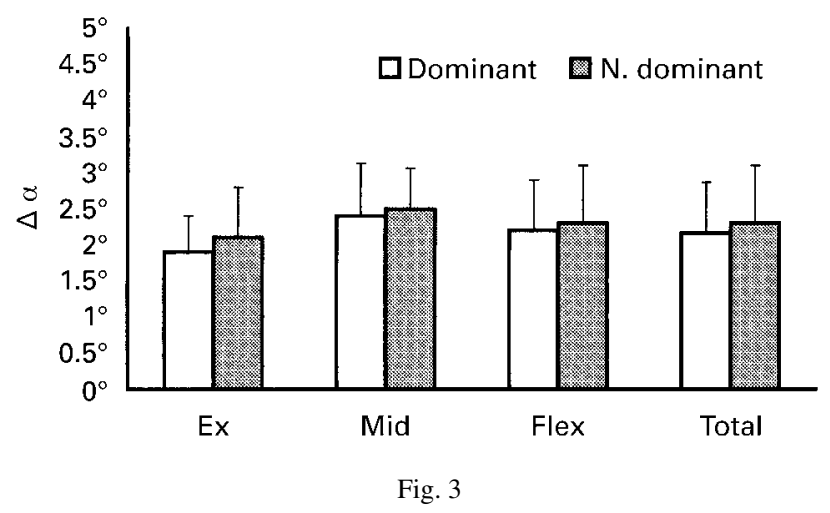

For the control group there were no statistical significant differences in the mean angle deviations between the dominant and the non-dominant sides ( $\Delta \alpha$, mean angle deviation; EX, extension interval $0^{\circ}$ to $20^{\circ}$; MID, midrange position $40^{\circ}$ to $60^{\circ}$; FLEX, flexion interval $80^{\circ}$ to $100^{\circ}$ ).

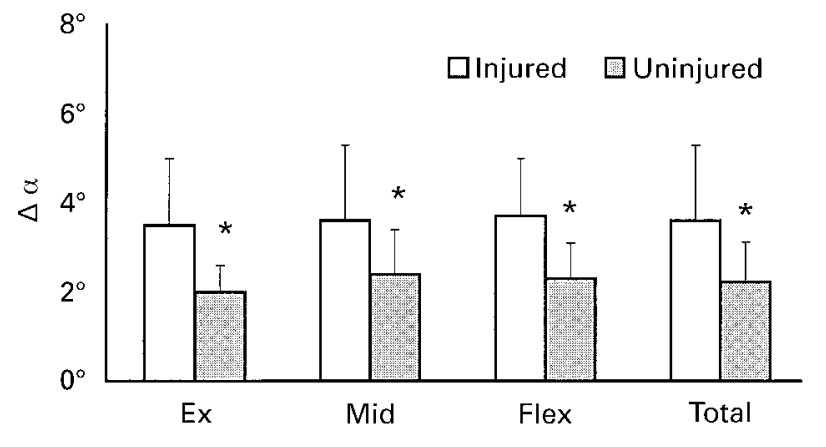

Fig. 5

Preoperative testing of patients with chronic instability showed persisting significant differences of the angle deviations between both sides at all testing intervals (for abbreviations see Figure 3).

points. All had a standardised clinical examination which did not reveal any disorder or history of knee injury.

Operative technique. In nine patients we used the open technique with a limited medial arthrotomy after arthroscopy and the necessary meniscal surgery. In 11 patients, standard arthroscopic reconstruction of the ACL was undertaken. In all patients, an ipsilateral patellar tendon autograft was used. Postoperative treatment included partial weightbearing for three weeks, no brace and closed chain exercises.

Measurement of proprioception. This was measured using the method described by Barrett ${ }^{1}$ (Fig. 2) which determines the reproduction of passive positioning of the knee at a constant angle velocity of $0.5 \%$. All external clues to the position or movement of the limb were excluded, except those from the knee. Subjects were seated in a $60^{\circ}$ reclining position to encourage relaxation and blindfolded. Their legs were allowed to hang freely over the side of the seat. A pneumatic cuff was connected to the mobile arm of the apparatus applied below the knee. The device allowed full passive flexion and extension without any varus or valgus deviation of the knee. The joint angle was recorded by a digital microprocessor with an accuracy of $0.1^{\circ}$. Each subject was given two test runs to become

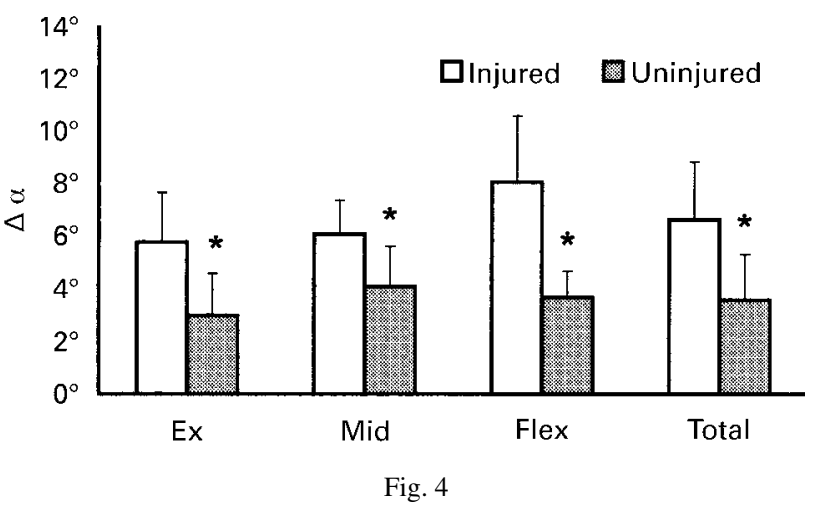

For the patients with acute instability, there was a significant increase in the angle deviations of the injured knee at all testing intervals. The uninjured side revealed a smaller, but also significant, increase of the angle deviations (for abbreviations see Figure 3).

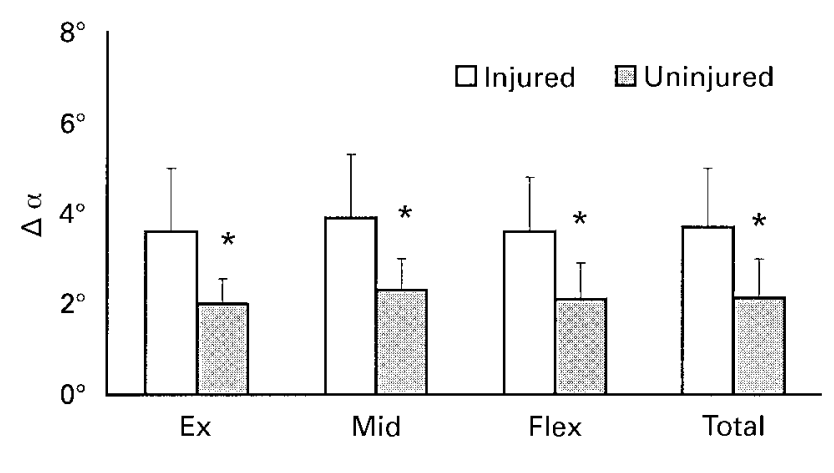

Fig. 6

Three months after operation the patients with chronic instability showed a slight increase of the angle deviations compared with the preoperative testing (for abbreviations see Figure 3).

familiar with the procedure. The reproduction of passive positioning was measured by asking the patient to reproduce passively a randomised reference position which was given by the apparatus in any of the testing intervals (extension, $0^{\circ}$ to $20^{\circ}$; mid-range position, $40^{\circ}$ to $60^{\circ}$; flexion, $80^{\circ}$ to $100^{\circ}$ ). Proprioception was determined as the difference between the reference position given by the examiner and the angle set by the patient with the angle deviation of the healthy knee taken as the normal value. The starting position of each measurement was $0^{\circ}$. The sequence of testing intervals was randomised as was the knee which was first tested.

All patients were assessed by testing proprioception evaluation by the Lysholm knee score, and the ligaments using the KT-1000 arthrometer (MEDmetric Corporation, San Diego, California) with maximum manual force in $30^{\circ}$ of flexion. Assessment of the patient's satisfaction was by a visual analogue scale graded from ten (excellent) to one (poor). The correlation between proprioception, patient satisfaction, ligament laxity and the Lysholm score was recorded.

Routine statistical analysis was carried out using the paired/unpaired Student $t$-test with a level of significance of $\mathrm{p}<0.05$, and by Pearson correlation analysis. 
$804 \quad$ R. W. FREMEREY, P. LOBENHOFFER, J. ZEICHEN, M. SKUTEK, U. BOSCH, H. TSCHERNE

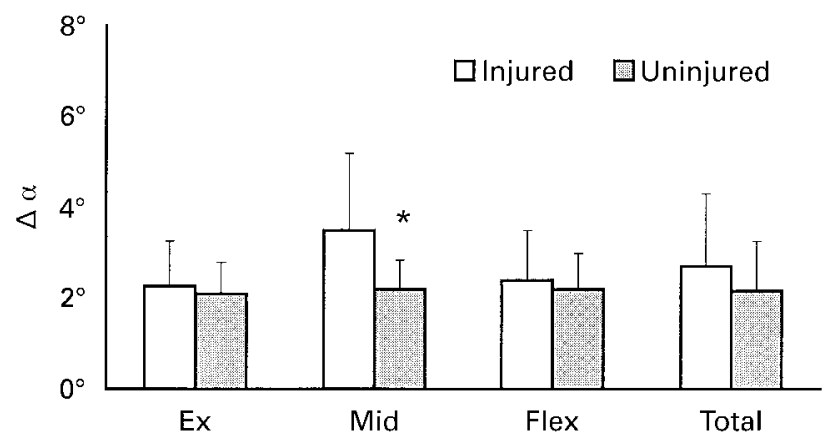

Fig. 7

Six months after operation the angle deviations in both the extension and flexion interval became normal in the patients with chronic instability. In the mid-range position, the significant difference between both sides persisted (for abbreviations see Figure 3).

\section{Results}

Control group. There was no significant difference between the dominant (angle deviation $2.2 \pm 0.7^{\circ}$ ) and the non-dominant sides $\left(2.3 \pm 0.8^{\circ}\right)$. The lowest angle deviation was assessed in the extension interval $\left(0^{\circ}\right.$ to $\left.20^{\circ}\right)$ at 1.9 $\pm 0.5^{\circ}$ (dominant) and $2.1 \pm 0.7^{\circ}$ (non-dominant). The deviations measured in the flexion interval $\left(80^{\circ}\right.$ to $\left.100^{\circ}\right)$ were slightly but not significantly higher at $2.2 \pm 0.7^{\circ}$ (dominant) and $2.3 \pm 0.8^{\circ}$ (non-dominant). By contrast, the deviations found in the mid-range position $\left(40^{\circ}\right.$ to $\left.60^{\circ}\right)$ showed significantly higher deviations for both the dominant and the non-dominant sides compared with the data recorded in the extension and flexion intervals. There were no significant differences between women and men in any of the testing intervals (Fig. 3).

Patients with acute anterior knee instability. Acute trauma produced a gross increase in the angle deviations of the injured knees $\left(6.7 \pm 2.2^{\circ}\right)$. The healthy side also showed a significant increase $(\mathrm{p}<0.05)$ in the deviation $\left(3.6 \pm 1.8^{\circ}\right)$ as compared with the control group $\left(2.2 \pm 0.7^{\circ}\right)$. There was a significant difference $(\mathrm{p}<0.001)$ between the injured and the healthy side at all intervals. The lowest values of the injured side were measured in the extension interval (5.8 \pm $\left.1.9^{\circ}\right)$ and the highest deviation was found in the flexion interval $\left(8.1 \pm 2.5^{\circ}\right)$. On the healthy side, there were no significant differences in the deviations between the testing intervals (Fig. 4).

Patients with chronic anterior knee instability. The preoperative testing was carried out on the day of admission (mean, $12.4 \pm 3.7$ months after trauma). All patients were free from pain, with every knee having a full range of movement. Compared with the patients with acute trauma $\left(6.7 \pm 2.2^{\circ}\right)$, the injured side revealed a significant $(\mathrm{p}<0.001)$ mean decrease of the angle deviation of $3.6 \pm$ $1.7^{\circ}$. The healthy side showed decreased deviations, but with no significant difference $(\mathrm{p}<0.05)$ compared with the healthy volunteers. There was a significantly higher deviation $(\mathrm{p}<0.001)$ on the injured side than on the non-injured

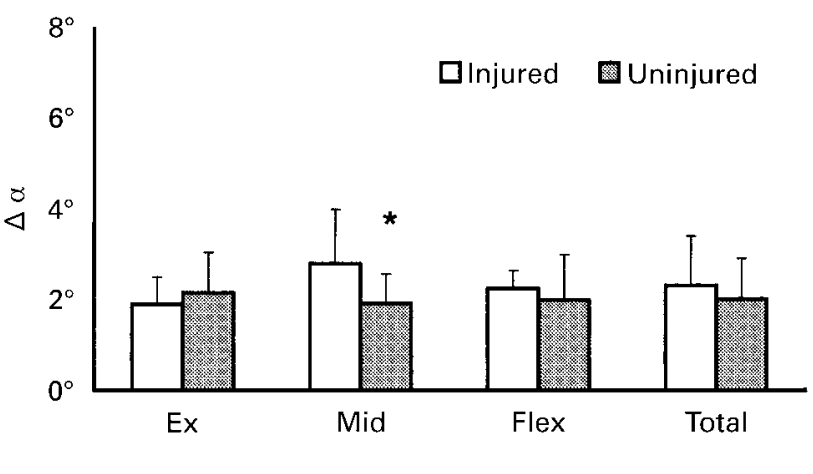

Fig. 8

At the final follow-up the patients with chronic instability showed a further decrease of the angle deviations in the mid-range position, but there was still a significant difference between both sides at this particular testing interval. There is persisting restitution of proprioception in the extreme positions (for abbreviations see Figure 3 ).

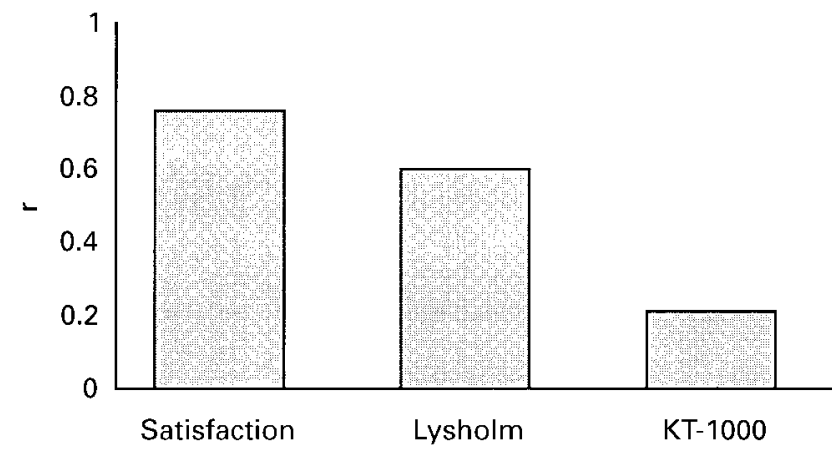

Fig. 9

At the final follow-up, in patients with chronic instability, the highest correlation was found between patient satisfaction and proprioception $(\mathrm{r}=0.76)$ and the lowest correlation was between proprioception and ligament laxity $(\mathrm{r}=0.21)$.

side at all of the testing intervals. The injured knees showed the lowest angle deviation of $3.5 \pm 1.5^{\circ}$ in the extension interval (Fig. 5).

After three months of intensive rehabilitation we found a slightly higher angle deviation on the injured side $(3.7 \pm$ $1.3^{\circ}$ ) compared with the preoperative data. The differences between the injured and uninjured sides were significant at any testing interval $(\mathrm{p}<0.001)$, and thus there was no improvement in proprioception after three months. The deviations measured on the healthy side were similar to the preoperative values (Fig. 6).

After six months of rehabilitation and a return to normal activities of daily living we found a significant $(\mathrm{p}<0.001)$ decrease in the angle deviations of the injured side $(2.7 \pm$ $\left.1.6^{\circ}\right)$. There were no significant differences of the deviations measured in both the extension $\left(2.3 \pm 1.0^{\circ} v 2.1 \pm\right.$ $\left.0.7^{\circ}\right)$ and the flexion interval $\left(2.4 \pm 1.1^{\circ} v 2.2 \pm 0.8^{\circ}\right)$. The angle deviations assessed in the mid-range position did not reach the values of the healthy side $(\mathrm{p}<0.001)$, and again, there was no effect on the healthy side (Fig. 7).

At the final follow-up $(3.7 \pm 0.3$ years). Compared with the examination six months after ACL reconstruction, there 
was a significant decrease of the angle deviations of the injured side in the mid-range position $\left(3.5 \pm 1.7^{\circ} \vee 2.8 \pm\right.$ $\left.1.2^{\circ}, \mathrm{p}<0.01\right)$, but the difference between the injured $(2.8$ $\left.\pm 1.2^{\circ}\right)$ and the uninjured sides $\left(1.9 \pm 0.7^{\circ}\right)$ was still significant $(\mathrm{p}<0.01)$. In the extension interval, there was even a slight, but not significantly lower angle deviation on the injured side compared with the uninjured knee $1.9 \pm$ $\left.0.6^{\circ} v 2.2 \pm 0.9^{\circ}, \mathrm{p}=0.067\right)$. In the flexion interval, there were also no significant differences between the two sides documented (Fig. 8).

Development of proprioception. Acute trauma caused gross damage to proprioception with an increase in the mean angle deviation of up to $6.7 \pm 2.2^{\circ}$ on the injured side and also a smaller, but still significant, increase in the values of the uninjured side compared with the healthy volunteers. After preoperative rehabilitation the angle deviations of the injured side decreased significantly, but did not reach the values of the uninjured side. In patients with knee instability a slight increase in the angle deviations was found on the injured side three months after operation. After six months, however, the values in both the flexion and the extension interval had become normal. By contrast, no recovery could be found in the mid-range position. At the final examination, there was a further decrease in the angle deviation measured in the mid-range position, but it did not reach the value of the uninjured side. The normal values in both the extension and flexion interval, which had been documented at six months after operation, remained.

Limited arthrotomy versus standard arthroscopic ACLreconstruction. Three months after operation, patients with a limited arthrotomy showed slightly, but not significantly, higher deviations $\left(3.8 \pm 1.3^{\circ}\right)$ on the injured side compared with the arthroscopically treated patients $\left(3.6 \pm 1.5^{\circ}\right)$. After six months and at final follow-up $(3.7 \pm 0.3$ years $)$, there were no statistically significant differences in the deviations found in any of the testing intervals.

Correlations. At final follow-up the mean Lysholm score was $93.3 \pm 4.7$ points, the mean score for patient satisfaction was $8.5 \pm 0.6$ points, and the mean difference of the values for maximum manual force between both sides was $3.1 \pm 2.3 \mathrm{~mm}$. The highest correlation was found between proprioception and patient satisfaction $(\mathrm{r}=0.76)$; that between proprioception and the Lysholm score was $r=0.60$ and between patient satisfaction and Lysholm score $r=0.38$. The lowest values were found between proprioception and ligament laxity $(r=0.21)$ and between patient satisfaction and ligament laxity $(r=0.18)($ Fig. 9).

\section{Discussion}

Like other authors ${ }^{1,6,7}$ we could not find differences in proprioception between the dominant and non-dominant side or between women and men. Only Sell, Zacher and Lack $^{8}$ were able to show increased values in men when compared with women.
In the healthy volunteers, the best results were recorded in the flexion and extension interval when the ligaments and capsule are more stretched than in mid-range. Extensive stretching stimulates the slowly-adapting Ruffini and Golgi receptors ${ }^{9}$ which boost proprioceptive awareness. This correlates with clinical experience that proprioceptive skills may play a major role in protecting the knee, especially at the extreme ranges of movement. ${ }^{6}$ The assessment of proprioception by using the test of reproduction of passive positioning is a valid and established method described by Barrett. ${ }^{1}$

Acute rupture of the ACL caused a gross increase in the mean angle deviations on the injured leg and also a lower, but still significant, loss of proprioception on the healthy side at all the testing intervals. The fact that injury leads to a deterioration of proprioception has been documented in experimental studies by Kennedy, Alexander and Hayes ${ }^{10}$ who described a direct inhibition of the quadriceps caused by joint effusion and capsular distension.

There are many studies which report reduced proprioception after rupture of the ACL, ${ }^{5,11}$ but as we know of none which have examined longitudinally the recovery of proprioception after treatment. After the programme of preoperative rehabilitation, we found a significant decrease in the angle deviations compared with those of the trauma group, but the values of the healthy side could not be reached in any of the testing intervals. This proprioceptive deficit in chronic ACL-deficient knees may be the result of a loss of passive restraint which causes stretching of capsular structures and a decreased response of capsular receptors. The disturbance of proprioception may also be a cause of increased joint laxity as well as a result of it.

Three months after ACL reconstruction, there was a slight, but not significant, deterioration of proprioception compared with that before operation.

Six months after operation, restitution of proprioception was documented in both the flexion and the extension interval, but a deficit of proprioception persisted in the midrange position, indicating that restoration was achieved by the combination of reconstruction and rehabilitation only at the extreme ranges of movement. At the final follow-up ( $3.7 \pm 0.3$ years), there was further improvement of proprioception especially in the mid-range position compared with the examination six months after operation, but full restitution of proprioception was again not achieved. The ACL-deficient knee moves along a non-physiological axis $^{12}$ which causes disturbance of cortical interpretation as well as damage to the remaining receptors in the capsule and ligaments due to increased laxity. By restoring mechanical stability by ACL reconstruction, these remaining receptors seem to recover and to compensate for the lack of mechanoreceptors in the ACL, especially at the extreme ranges of movement. By restoring a more physiological joint movement, cortical interpretation is enhanced. ${ }^{1}$ This biological process seems to start relatively early after reconstruction of the ACL so that complete functional 
recovery, documented at the extremes of movement, occurred between three and six months after operation. During the period between six months and $3.7 \pm 0.3$ years after ACL reconstruction, proprioception continued to improve especially in the mid-range position, which may be due to further recovery of undamaged receptors or to a regrowth of neurones. Proprioception did not recover completely, however, in the mid-range position.

MacDonald et $\mathrm{al}^{13}$ reported reduced proprioception after ACL reconstruction. Their study included eight patients with hamstring reconstruction, with a mean follow-up of 24 months, and eight with patellar tendon reconstruction with a mean follow-up of 31 months. The testing was carried out using the $30^{\circ}$ to $40^{\circ}$ interval of knee flexion. In line with our results, they reported a persistent lack of proprioception in the mid-range position.

$\mathrm{Co}$ et $\mathrm{al}^{7}$ reported recovery of proprioception after ACL reconstruction in ten patients with patellar tendon reconstruction and a mean follow-up of 31.6 months. The testing interval which they used was between $40^{\circ}$ of knee flexion and full extension, which is similar to the extension interval used in our study.

We confirm the view that, after ACL reconstruction, clinical assessment of ligament tightness as well as the Lysholm score correlated poorly with patient satisfaction. By contrast, there was a high correlation between the latter and proprioception which confirms its clinical relevance. The relationship between proprioception and the subjective feeling of stability has already been demonstrated. ${ }^{1}$ Patients with chronic knee injuries often report the beneficial effect of a bandage, although this provides no biomechanical support, but an increase in proprioception by stimulation of cutaneous receptors.

Our study has shown that most of the proprioceptive recovery occurred between three and six months after ACL reconstruction with further improvement after that period in the mid-range position. These results are clinically important because they support the concept that the patient can be allowed to return gradually to his previous level of activity after three to four months. An earlier return to full activity could be dangerous due to the proprioceptive deficit still present at this time. However at this time the graft may still be relatively weak, as has been demonstrated in the posterior cruciate ligament. ${ }^{14}$ This difference in time between the partial restitution of proprioception and the healing of the graft should be considered when planning rehabilitation.

No benefits in any form have been received or will be received from a commercial party related directly or indirectly to the subject of this article.

\section{References}

1. Barrett DS. Proprioception and function after anterior cruciate reconstruction. J Bone Joint Surg [Br] 1991;73-B:833-7.

2. Grigg P, Hoffmann AH. Properties of Ruffini afferents revealed by stress analysis of isolated sections of cat knee capsule. J Neurophysiol 1982;47:41-54.

3. Schultz RA, Miller DC, Kerr CS, Micheli L. Mechanoreceptors in human cruciate ligaments: a histological study. J Bone Joint Surg [Am] 1984;66-A:1072-6.

4. Biedert RM, Stauffer E, Freiderich NF. Occurrence of free nerve endings in the soft tissue of the knee joint: a histologic investigation. Am J Sports Med 1992;4:430-3.

5. Beard DJ, Kyberd PJ, Fergusson CM, Dodd CAF. Proprioception after rupture of the anterior cruciate ligament: an objective indication of the need for surgery? J Bone Joint Surg [Br] 1993;75-B:311-5.

6. Barrack RL, Skinner HB, Brunet ME, Cook SD. Joint kinesthesia in the highly trained knee. J Sports Med Phys Fitness 1984;24:18-20.

7. Co FH, Skinner HB, Cannon WD. Effect of reconstruction of the anterior cruciate ligament on proprioception of the knee and the heel strike transient. J Orthop Res 1993;11:696-704.

8. Sell S, Zacher J, Lack S. Disorders of proprioception of the arthrotic knee joint. Z Rheumatol 1993;52:150-5.

9. Johansson H, Sjölander P, Sojka P. A sensory role for the cruciate ligaments. Clin Orthop 1991;268:161-78.

10. Kennedy JC, Alexander IJ, Hayes KC. Nerve supply of the human knee and its functional importance. Am J Sports Med 1982;10:329-35.

11. Jerosch J, Prymka M. Knee joint proprioception in normal volunteers and patients with anterior cruciate ligament tears, taking special account of the effect of a knee bandage. Arch Orthop Trauma Surg 1996;115:162-6

12. Berchuck M, Andriacchi TP, Bach BR, Reider B. Gait adaptations by patients who have a deficient anterior cruciate ligament. J Bone Joint Surg [Am] 1990;72-A:871-7.

13. MacDonald PB, Hedden D, Pacin O, Sutherland K. Proprioception in anterior cruciate ligament-deficient and reconstructed knees. Am J Sports Med 1996;24:774-8.

14. Bosch U, Kasperczyk WJ. Healing of the patellar tendon autograft after posterior cruciate ligament reconstruction: a process of ligamentisation? An experimental study in a sheep model. Am J Sports Med 1992;20:558-66. 